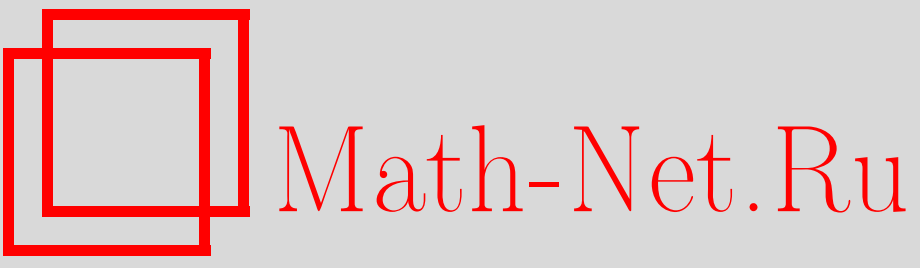

Р. П. Кузьмина, О решении уравнения Ван-дер-Поля, УМН, 1997, том 52, выпуск 1, 231-232

DOI: https://doi.org/10.4213/rm812

Использование Общероссийского математического портала Math-Net.Ru подразумевает, что вы прочитали и согласны с пользовательским соглашением

http: //www.mathnet.ru/rus/agreement

Параметры загрузки:

IP : 54.198 .55 .26

26 апреля 2023 г., 16:03:41

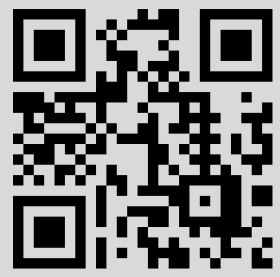




\section{О РЕШЕНИИ УРАВНЕНИЯ ВАН-ДЕР-ПОЛЯ}

Р. П. КУЗьминА

Рассмотрим уравнение Ван-дер-Поля

$$
\ddot{z}=-z+\varepsilon\left(1-z^{2}\right) \dot{z}
$$

с заданными начальными значениями $z$ и $\dot{z}$. Здесь точка означает дифференцирование по независимой переменной $\tau$. На интервале времени $\tau$ порядка $\varepsilon^{-1}$ задача Коши для уравнения (1) является сингулярно возмущенной и метод Пуанкаре к ней не применим. Переходом к переменHLIM

$$
a=\sqrt{z^{2}+(\dot{z})^{2}}, \alpha=\arg (z+i \dot{z})
$$

уравнение (1) приводится к стандартной для применения метода осреднения форме [1]. Рассмотрим другой подход к решению задачи (1), а именно: приведем (1) к почти регулярной задаче Коши [2]

$$
\frac{d x}{d t}=F(x, t, \varepsilon, f(t, \varepsilon)),\left.\quad x\right|_{t=0}=0
$$

где $f$ - гладкая и ограниченная функция при малшх значениях $\varepsilon>0, F(x, t, \varepsilon, f)$ - гладкая функция при малшх значениях $\varepsilon \geqslant 0$. Для этого введем новые переменные

$$
\begin{gathered}
x_{1}=a-v-e g_{1}, \quad x_{2}=\alpha-\varphi-e g_{2}, \quad \tau=t / \varepsilon, \\
g_{1}=\frac{a}{4} \sin 2 \alpha-\frac{a^{3}}{32} \sin 4 \alpha+C_{1}, \quad g_{2}=\frac{1}{2} \cos ^{2} \alpha-\frac{a^{2}}{4} \cos ^{4} \alpha+C_{2}, \\
v=2\left[1+C_{0} \exp (-t)\right]^{-1 / 2}, \quad \varphi=-t / \varepsilon+\alpha_{0}, C_{0}=4 a_{0}^{-2}-1, \\
C_{1}=-\frac{a_{0}}{4} \sin 2 \alpha_{0}+\frac{a_{0}^{3}}{32} \sin 4 \alpha_{0}, \quad C_{2}=-\frac{1}{2} \cos ^{2} \alpha_{0}+\frac{a_{0}^{2}}{4} \cos ^{4} \alpha_{0} .
\end{gathered}
$$

Здесь $a_{0}, \alpha_{0}$ - начальные значения переменных (2). Задача Коши для $x_{1}, x_{2}$ имеет вид

$$
\begin{gathered}
\frac{d x_{1}}{d t}=\frac{a}{2}\left(1-\frac{a^{2}}{4}\right)-\frac{v}{2}\left(1-\frac{v^{2}}{4}\right)+\varepsilon h_{1}, \quad \frac{d x_{2}}{d t}=\varepsilon h_{2}, \\
\left.x_{1}\right|_{t=0}=\left.x_{2}\right|_{t=0}=0 .
\end{gathered}
$$

Здесь

$$
\begin{aligned}
& h_{1}=-a \sin \alpha \cos \alpha\left(1-a^{2} \cos ^{2} \alpha\right)\left[\frac{1}{2} \cos ^{2} \alpha+\frac{a^{2}}{8}\left(2-\cos ^{2} \alpha-2 \cos ^{4} \alpha\right)\right], \\
& h_{2}=\sin ^{2} \alpha \cos ^{2} \alpha\left(1-a^{2} \cos ^{2} \alpha\right)\left(1-\frac{a^{2}}{2} \cos ^{2} \alpha\right) .
\end{aligned}
$$

Зависимость $a, \alpha$ от $x_{1}, x_{2}, t, \varepsilon$ определяется формулами (4). Из них следует система уравнений

$$
\begin{gathered}
x_{1}=a-v-\varepsilon g_{1}, \quad \cos \varphi=\cos \alpha \cos \left(x_{2}+\varepsilon g_{2}\right)+\sin \alpha \sin \left(x_{2}+\varepsilon g_{2}\right), \\
\sin \varphi=-\cos \alpha \sin \left(x_{2}+\varepsilon g_{2}\right)+\sin \alpha \cos \left(x_{2}+\varepsilon g_{2}\right) .
\end{gathered}
$$

Исследуя (5), (6), нетрудно получить:

1) (5) - почти регулярная задача Коши (3) со значениями $x=\left(x_{1}, x_{2}\right), f=(\cos \varphi, \sin \varphi)$,

$$
U(t, s)=\left(\begin{array}{cc}
q(t, s) & 0 \\
0 & 1
\end{array}\right), \quad q(t, s)=\exp (-t+s)\left[\frac{1+C_{0} \exp (-s)}{1+C_{0} \exp (-t)}\right]^{3 / 2},
$$


$(U(t, s)$ - матрица Коши уравнений в вариациях, см. [2]),

2 ) решение задачи (5) можно построить в виде ряда

$$
x(t, \varepsilon)=\sum_{k=0}^{\infty} y^{(k)}(t, \varepsilon) \varepsilon^{k},
$$

где $y^{(k)}(t, \mu)$ - коэффициенты разложения в ряд решения регулярно возмушенной задачи Коши

$$
\frac{d y}{d t}=F(y, t, \varepsilon, f(t, \mu)),\left.\quad y\right|_{t=0}=0
$$

$y(t, \varepsilon, \mu)=\sum_{k=0}^{\infty} y^{(k)}(t, \mu) \varepsilon^{k}$,

3 ) для задачи (5) справедливы теоремы $1,3,5,7,9$ из [2]. Из этих теорем следует, что для любых значений $T>0, \beta, 0 \leqslant \beta<1 / 2, n \geqslant 0$ найдутся $\varepsilon_{*}>0, C_{*}, C_{*}^{\circ} \geqslant 0$, не зависящие от $t, \varepsilon$ и такие, что при $0 \leqslant t \leqslant T \varepsilon^{-\beta}, 0<\varepsilon \leqslant \varepsilon_{*}$ :

1) решение задачи (5) существует, единственно, удовлетворяет неравенству $\| x(t, \varepsilon)-$ $X_{n}(t, \varepsilon) \| \leqslant \varepsilon^{n+1} t\left(C_{*}^{\circ} t^{2 n}+C_{*}\right)$, где $X_{n}(t, \varepsilon)$ - частичная сумма ряда $(7)$,

2) ряд (7) сходится равномерно к решению задачи (5).

$\mathrm{K}$ задаче Коши для остаточного члена $u=x-X_{1}(t, \varepsilon)$ был применен алгоритм доказательства теоремы 2.1 в работе [3]. Оценка остаточного члена $u$ и интервала существования решения задачи $(5)$ при этом улучшилась. Кроме того, в $X_{1}(t, \varepsilon)$ были выделены члены порядка $O(\varepsilon)$ : $X_{1}=\bar{X}_{1}+\Delta X_{1},\left\|\Delta X_{1}\right\| \leqslant C \varepsilon^{2}$ при $t \geqslant 0, \varepsilon>0$. Наконец, полученные результаты были пересчитаны для исходной переменной.

РезУльтаты. Найдутся такие значения $T>0, \varepsilon_{*}>0$ и постоянные $K$, что при $0 \leqslant \tau \leqslant T \varepsilon^{-2}, 0<\varepsilon \leqslant \varepsilon_{*}$ решение задачи Коши для уравнения (1) существует, единственно и удовлетворяет неравенствам

$$
\begin{gathered}
|z-\bar{a} \cos \bar{\alpha}| \leqslant \varepsilon^{2}\left(K \varepsilon^{2} \tau^{2}+K\right), \quad|a-\bar{a}| \leqslant \varepsilon^{2}(K \varepsilon \tau+K), \quad|\alpha-\bar{\alpha}| \leqslant \varepsilon^{2}\left(K \varepsilon^{2} \tau^{2}+K\right), \\
\bar{a}=v+\varepsilon C_{3} v\left(4-v^{2}\right)+\frac{\varepsilon v}{4} \sin 2 \varphi\left(1-\frac{v^{2}}{4} \cos 2 \varphi\right), \\
\bar{\alpha}=\varphi+\frac{\varepsilon t}{16}+\varepsilon\left[\frac{\ln v}{8}-\frac{5 v^{2}}{64}+\frac{\cos ^{2} \varphi}{2}\left(1-\frac{v^{2}}{2} \cos ^{2} \varphi\right)+C_{4}\right], \\
v=2\left[1+C_{0} \exp (-\varepsilon \tau)\right]^{-1 / 2}, \quad \varphi=-\tau+\alpha_{0}, \\
C_{3}=-\frac{\sin 2 \alpha_{0}}{4\left(4-a_{0}^{2}\right)}\left(1-\frac{a_{0}^{2}}{4} \cos 2 \alpha_{0}\right), \\
C_{4}=-\frac{\ln a_{0}}{8}+\frac{5 a_{0}^{2}}{64}-\frac{\cos ^{2} \alpha_{0}}{2}\left(1-\frac{a_{0}^{2}}{2} \cos ^{2} \alpha_{0}\right) .
\end{gathered}
$$

ЗАмечАниЕ. Нетрудно доказать существование периодического решения уравнения (1) и получить соответствуюшие асимптотические формулы, используя гладкость решения и исследуя функции $\bar{a}, \bar{\alpha}$.

\section{СПИСОК ЛИТЕРАТУРЫ}

[1] Гребеников Е. А. Метод усреднения в прикладных задачах. М.: Наука, 1986. [2] Кузьмина Р. П. // УМН. 1995. Т. 50. № 4. С. 161-162. [3] Кузьмина Р. П. Методмалого параметра в регулярно возмущенной задаче Коши. М.: Изд-во МГУ, 1991.

Московский государственньй

Принято редколлегией университет им. М. В. Ломоносова

25.12 .1996 